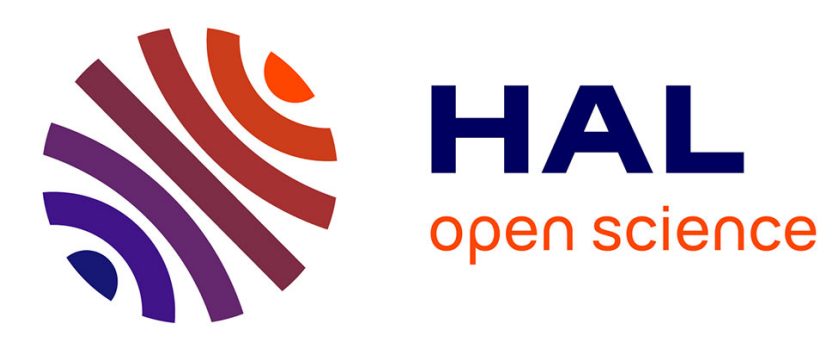

\title{
Autour de la table de George Sand: Éthique de la gourmandise sandienne
}

\author{
Amélie Calderone
}

\section{To cite this version:}

Amélie Calderone. Autour de la table de George Sand: Éthique de la gourmandise sandienne. Romantisme: la revue du dix-neuvième siècle, 2019, 186, pp.50-59. 10.3917/rom.186.0050 . halshs02404652

\section{HAL Id: halshs-02404652 \\ https://shs.hal.science/halshs-02404652}

Submitted on 17 Dec 2019

HAL is a multi-disciplinary open access archive for the deposit and dissemination of scientific research documents, whether they are published or not. The documents may come from teaching and research institutions in France or abroad, or from public or private research centers.
L'archive ouverte pluridisciplinaire HAL, est destinée au dépôt et à la diffusion de documents scientifiques de niveau recherche, publiés ou non, émanant des établissements d'enseignement et de recherche français ou étrangers, des laboratoires publics ou privés. 


\title{
Autour de la table de George Sand: \\ Éthique de la gourmandise sandienne
}

\author{
Amélie Calderone \\ [Version auteur]
}

\begin{abstract}
L'homme ne doit pas se retirer les nobles jouissances, et respirer à toute heure le parfum des mousses ou des genêts vaut encore mieux que de satisfaire un instant la gourmandise ${ }^{1}$.
\end{abstract}

« J'ai toujours mangé vite et en pensant à autre chose ${ }^{2}$ », confie George Sand dans Histoire de ma vie. Une telle déclaration pourrait laisser penser que la romancière néglige les plaisirs de la table, ce qu'un parcours de ses écrits personnels dément. Si la petite Aurore dévorait des friandises « en cachette ${ }^{3}$ », la jeune Sand avoue que «[s]on suprême bonheur est manger beaucoup 4 ", tandis que la "bonne dame de Nohant» produira son propre mythe, entretenu par la postérité5, d'organisatrice de festins, de cuisinière ou de faiseuse de confitures. Sand savait apprécier la bonne chère et ses œuvres regorgent de descriptions de repas ou d'appréciations quant aux usages alimentaires. Le rapport à la nourriture occupe ainsi une place plus centrale qu'il n'y paraît au sein de l'imaginaire sandien - constat qui pourrait paraître étonnant au regard de l'idéalisme supposé de l'auteur. Dans les méandres d'une écriture qui pense dans son mouvement, pourraient dès lors se dessiner les contours d'une philosophie au sens large de la gourmandise, laquelle mettrait à mal cette assignation à l'idéalisme dont la romancière a été victime, lieu commun déjà en son temps ${ }^{6}$. Circuler dans l'ensemble des textes sandiens - fiction, autobiographie et épistolaire confondus -, permettra d'interroger de façon critique la permanence supposée du discours sandien de et sur la gourmandise, et de mettre au jour les modulations dans le temps ainsi que sur les scènes publiques et privées, d'un imaginaire s'élaborant sur la longue durée au sein de supports d'écriture hétérogènes.

Reste que, si l'on considère, à la suite du Grand Larousse du XIXe siècle, que la gourmandise est le « vice de celui qui est gourmand», de celui qui « mange avec avidité

\footnotetext{
${ }^{1}$ George Sand, Monsieur Sylvestre, Paris, Michel Lévy frères, 1866, XIV, de Pierre à Philippe, p. 70.

2 George Sand, Histoire de ma vie, dans OEuvres autobiographiques, George Lubin (éd.), Paris, Gallimard, coll. « Bibliothèque de la Pléiade », 2 vol., 1970 et 1971, vol. I, III e partie, chap. 2, p. 667.

${ }^{3}$ Ibid., III partie, chap. 13, p. 938.

${ }^{4}$ George Sand, Correspondance, Georges Lubin (éd.), Paris, Garnier, coll. "Classiques Garnier », 25 vol., 1964-1991 ; vol. 26, Tusson, Du Lérot, 1995, vol. I, à Louis-Nicolas Caron, mardi soir [22] ou mercredi matin [23 juin 1824], p. 140.

5 Voir Christine Sand, À la table de George Sand, Paris, Flammarion, 1987; George Sand, Scènes gourmandes, repas et recettes du Berry, Béatrice Didier (éd.), Paris, EJL, 1999.

6 Voir, à ce sujet, Damien Zanone (dir.), George Sand et l'idéal. Une recherche en écriture, Paris, Honoré Champion, coll. "Littérature et Genre », 2017 ; et Naomi Schor, George Sand and idealism, New-York, Columbia University Press, 1993.
} 
et avec excès ${ }^{7} »$ - définition contenant une réprobation morale implicite -, il n'est pas certain que le terme convienne à George Sand, qui redéfinit la notion pour créer une gourmandise éthique. S'il lui arrive d'endosser un rôle de moralisatrice et de condamner un vice présenté selon certains topoï de son époque, elle rend néanmoins le penchant acceptable: maîtrisée et rationnalisée, la gourmandise est, sur le plan individuel, la garantie d'un plein exercice de la liberté d'esprit, tandis que sur le plan collectif, elle revêt un aspect politique, en ce qu'elle est fédératrice d'une communauté. À cet égard, elle apparaît comme l'une de ces realia susceptibles de penser l'idéal - en l'occurrence, l'utopie républicaine et sociale.

\section{Imaginaire sandien des nourritures terrestres : être ou ne pas être gourmand?}

Lorsque l'on sait combien le XIXe siècle associe la gourmandise à l'enfance ${ }^{8}$, l'on ne s'étonne pas de voir George Sand souscrire aux usages éducatifs de son époque avec son fils. À maintes reprises, ses lettres sont l'occasion de gourmander Maurice au sujet de ce que l'expression populaire condamne comme un "vilain défaut » qui doit rester l'apanage des jeunes années :

Tu as le défaut d'être un peu gourmand. Dans un enfant, ce n'est rien. On en rit. Mais chez un jeune homme, cela devient ridicule, dans un homme fait cela devient dégoûtant ${ }^{9}$.

Plus on avance en âge, plus la gourmandise semble devenir répréhenssible aux yeux d'une George Sand entendant conformer son fils à un état "adulte » qui est aussi celui d'une humanité civilisée. La gourmandise devient alors dé-goût et charrie avec elle tout un imaginaire corporel rendu à ses dimensions les plus viles et basses. Moralement condamnable, elle est un vice, terme en lequel se joue la laïcisation de la dimension catholique du péché capital de gourmandise. Le double cadre de l'intimité et du dessein éducatif d'une jeune mère dévoile une George Sand profondément marquée par son éducation.

Pourtant, l'œuvre publique de George Sand insiste moins sur la dimension abjecte de la gourmandise qu'elle n'exploite son potentiel comique. En atteste la figure récurrente du moine gourmand. L'univers romanesque de George Sand foisonne de ces protagonistes rabelaisiens, depuis le curé de Teverino - que Léonce convainc de se joindre à lui et Sabina en lui promettant un «excellent vin et des vivres assez recherchées ${ }^{10}$ »-, jusqu'au frère Cyprien de La Daniella - qui prend "grand plaisir à manger ${ }^{11} »-$, en passant par le «bon carme ${ }^{12}$ » qui occupe jusqu'aux dernières lignes des Maîtres sonneurs, dont la gourmandise est patente dès son entrée dans le roman :

\footnotetext{
${ }^{7}$ Pierre Larousse, Grand Dictionnaire universel du XIX ${ }^{e}$ siècle, Paris, Administration du Grand Dictionnaire Universel, t. 8, 1872, articles « Gourmandise » et « Gourmand », p. 1397.

${ }^{8}$ Voir, dans ce numéro, la contribution de Francis Marcoin.

${ }^{9}$ George Sand, Correspondance, éd. citée, vol. III, à Maurice Dudevand, 8 juillet 1836, La Châtre, p. 464.

10 George Sand, Teverino, dans Vies d'artistes, Marie-Madeleine Fragonard (éd.), Paris, Omnibus, 2004, p. 579.

${ }^{11}$ George Sand, La Daniella, Alex Lascar (éd.), Paris, Honoré Champion, 2016, chap. XXXI, p. 388.

12 George Sand, Les Maîtres sonneurs, Pierre Salomon et Jean Mallion (éd.), Paris, Classiques Garnier, 2014, Trente-deuxième veillée, p. 479.
} 
Il y vint même un frère quêteur, qui était de passage, et qui, sous prétexte de mendier pour son couvent, remplit fort bien son estomac, et buvait aussi rude que bûcheux ou fendeux qu'il y eût ${ }^{13}[\ldots]$.

Bons (voire avides) mangeurs et gais buveurs, les clercs sandiens n'en sont pas moins sympathiques. Leur drolatique gourmandise participe d'une régulation plus vaste des comportements alimentaires se dressant au fil des œuvres - non sans emprunter à certains stéréotypes. Le solide appétit est signe de vigueur et de pleine santé, surtout chez les jeunes hommes. En témoigne Sylvinet dans La Petite Fadette, qui cesse de s'alimenter lorsqu'il devient malheureux. C'est alors de la viande qu'il convient de lui donner, comme le lui indique l'héroïne lorsqu'elle le guérit :

Je vas sortir, et vous vous lèverez, Sylvain, car vous n'avez plus la fièvre [...]. Vous mangerez ensuite ce que votre mère vous présentera de ma part. C'est de la viande, et je sais que vous vous en dites dégoûté, et que vous ne vivez plus que de mauvais herbages. Mais il n'importe, vous vous forcerez ${ }^{14}$ [...].

Le régime carné est à la fois réservé aux faibles et aux hommes. Les femmes, quant à elles, se doivent de manger avec modération, à l'instar de Consuelo qui ne déguste le festin de Mayer "qu'avec retenue ${ }^{15}$ », alors même qu'elle est affamée. En revanche, conformément aux lieux communs d'époque ${ }^{16}$, les protagonistes féminins tendent à être associées à une gourmandise sucrée, à ces "friandises que les dames ne dédaignent pas ${ }^{17}$ » comme le dit, dans La Comtesse de Rudolstadt, Schwartz à Consuelo, lorsque celle-ci réclame des repas plus substantiels que l'eau et le pain qu'on lui réserve... ainsi qu'un chocolat. L'héroïne sublime s'abandonne parfois, elle aussi, aux douceurs terrestres : "C'est plutôt ce délicieux café à la vénitienne dont j'ai pris deux tasses au lieu d'une, par pure gourmandise ${ }^{18}$ !» Daniella cède de même à des « habitudes de gâterie [toute] féminine ${ }^{19}$ » en veillant, par l'intermédiaire de Tartaglia, à ce que les plaisirs du palais soient bien prodigués à son amant, le narrateur Valreg :

[...] vous aurez du café succulent pour digérer, car la Daniella m’a dit: "Surtout, soigne-lui son café ; il n'a pas d'autre gourmandise ${ }^{20}$. »

Les femmes des romans sandiens se présentent volontiers comme des mères nourricières, figures maternelles archétypales au service des joies gustatives et de la santé des hommes - poncif qui éclaire l'élaboration par Sand de sa légende en bonne dame de Nohant cuisinière. Maîtresses dans l'art de confectionner les mets - songeons à la gouvernante du chanoine de Consuelo que le clerc conserve en dépit de ses défauts parce qu'elle « lui faisait de si bonnes confitures, et s'entendait si bien à conserver ses fruits, qu'il supportait sa méchante humeur $[\ldots]^{21} »-$, elles sont également les garantes de la force de vie des jeunes héros. Ainsi Brulette, qui «avait toujours un morceau de

${ }^{13}$ Ibid., Quinzième veillée, p. 216.

${ }^{14}$ George Sand, La Petite Fadette, Pierre Salomon et Jean Mallion (éd.), Paris, Classiques Garnier, 2014, chap. XXXIX, p. 263.

15 George Sand, Consuelo. La Comtesse de Rudolstadt, Damien Zanone (éd.), Paris, Robert Laffont, coll. «Bouquins », 2004, chap. LXX, p. 450.

${ }^{16}$ Jean-Anthelme Brillat-Savarin, Physiologie du goût, ou Méditations de gastronomie transcendante, Paris, Sautelet, vol. 1, 1826, p. 270 : «La gourmandise comprend aussi la friandise [...]. C'est une modification introduite en faveur des femmes [...].»

${ }^{17}$ George Sand, La Comtesse de Rudolstadt, éd. citée, chap. XIV, p. 860.

${ }^{18}$ George Sand, Consuelo, éd. citée, chap. LXXXI, p. 535.

${ }^{19}$ George Sand, La Daniella, éd. citée, chap. XXXI, p. 388.

${ }^{20} \mathrm{Idem}$.

${ }^{21}$ George Sand, Consuelo, éd. citée, chap. LXXVII, p. 510. 
viande en réserve [pour Joseph], quand il venait la voir, et, soit qu'il eût faim ou non, le lui faisait manger $[\ldots]^{22}$.»

Toutes ces realia mettent à mal le prétendu idéalisme de George Sand, qui ne se dégage pas de la matérialité, mais l'alimente par l'idée. L'auteur valorise en outre un comportement alimentaire aux antipodes de la gourmandise : le jeûne. Le cheminement des héros idéaux sandiens au sein du monde les conduit généralement vers une forme d'allègement, se manifestant dans leur rapport à la nourriture. L'on pense ici à Consuelo et Albert menant une rude vie de bohême au terme de leur parcours les conduisant à partager le "repas frugal ${ }^{23}$ » du pauvre. Bien des protagonistes tendent à une forme d'angélisme ou se voient assimilés à des oiseaux, animaux sandiens par excellence, loués pour leur ignorance de la gourmandise :

J'aime le chien, mais pas tous les chiens. [...] Leur gourmandise à tous me chagrine. [...] L'homme-chien n'est pas un beau type. Mais l'oiseau, je le soutiens, est l'être supérieur dans la création ${ }^{24}$.

Fins et éthérés, les oiseaux incarnent ici-bas l'aspiration sandienne au sublime et à l'idéal. Ils matérialisent la spiritualité. Et la gourmandise, entre vice ridicule, défaut véniel, réponse à une nécessité corporelle et jouissance terrestre, cristallise les va-etvient de George Sand entre matière et esprit: les comportements alimentaires contribuent au vaste mouvement qui consiste à tenter d'incarner la pensée. Dès lors, en tant que passion toujours soupçonnée d'asservir le ventre comme l'esprit, la gourmandise devient un moyen privilégié de penser la relation entre corps et âme.

\section{Mens sana in corpore sano : l'appel de la sobriété}

La gourmandise n'est pas simple réponse à la sensation de faim : elle est un audelà, qualitatif et quantitatif, au seul besoin, s'exprimant par le corps et s'imprimant en lui. C'est donc naturellement au nom de ses manifestations corporelles que Sand la proscrit dans un premier temps. L'excès de nourriture engendre un embonpoint qui répugne à l'écrivain - ce qu'elle expose dans l'intimité :

Je vous dirais que j'ai assisté avant hier à la bénédiction nuptiale de Duvernet avec une grosse grande large lourde, rouge, ronde, fille de 16 ans. Si elle a de l'appétit en proportion de ses dimensions, elle doit être une rude mangeuse ${ }^{25}[\ldots]$.

L'accumulation des adjectifs renforcée par l'allitération rugueuse en $R$ dénonce la force du sentiment de répulsion qui anime l'auteur. Comme si Sand avait peur que les qualités physiques de la mariée ne lui soient communiquées par contagion, elle « fui[t] la noce», alléguant qu'elle a «horreur de la mangeaille [...]». La gourmandise devient, étymologiquement, obscène : indécente dans son surcroît de visibilité.

Il est surprenant que celle qui s'affirme républicaine et socialiste puisse exprimer ce que notre regard a posteriori perçoit comme une vision de classe vis-à-vis des campagnards: ces discours d'un inavouable mépris sont naturellement bannis de l'œuvre publique. En outre, ils se feront plus rares dans les écrits privés avec la

\footnotetext{
22 George Sand, Les Maîtres sonneurs, éd. citée, Deuxième veillée, p. 36.

${ }^{23}$ George Sand, La Comtesse de Rudolstadt, éd. citée, Épilogue, p. 1136.

${ }^{24}$ George Sand, Histoire de ma vie, éd. citée, vol. I, Ière partie, chap. 1, p. 17.

25 George Sand, Correspondance, éd. citée, vol. II, à Émile Regnault, [Nohant, 29 août 1832], p. 150. Idem pour les deux citations qui suivent.
} 
maturité. Il ne faudrait cependant pas croire que ce sont des considérations esthétiques qui animent alors la jeune femme de lettres. D'un point de vue pratique, l'embonpoint pourrait nuire à l'agilité et à la robustesse physiques nécessaires au travail, comme elle l'écrit à son fils : «Le gourmand est [...] est lourd après avoir mangé et peu propre au travail ${ }^{26} . »$

Au-delà de ces entraves physiques, c'est parce que le plaisir - en l'occurrence, gustatif - n'a point de bornes qu'il est nuisible. L'homme qui se livre à son assouvissement exclusif est irrémédiablement conduit à l'oisiveté et à une quête du gain financier sans effort :

Il [au gourmand] lui faut donc du repos, et pour peu qu'il manque un peu d'appétit, il [...] se ruine en médicaments, sauf à se tuer plus tard d'indigestion. Un tel homme est capable de tout. Le manque d'argent étant ce qu'il redoute le plus, et l'épaississement de ses facultés ne lui permettant pas d'en gagner honorablement, il se vendra au premier venu, ou fera volontiers des actions de mauvaise foi $[\ldots]^{27}$.

En d'autres termes, le gourmand est condamné à l'immoralité. Et si la gourmandise n'est pas la cible d'une mise en accusation moralisatrice, elle fait en revanche l'objet d'une condamnation morale, énoncée dans une perspective sociale. Sa réprobation participe d'une entreprise plus vaste : celle de la critique de l'argent non gagné à la sueur de ses efforts, suspect d'être acquis malhonnêtement. Et dès lors qu'il s'agit de considérer la gourmandise sous son jour social, non seulement les écrits publics de Sand rejoignent ses affirmations proférées dans l'intimité, mais son discours se maintient dans le temps. C'est ainsi par la contamination du vocabulaire de la gourmandise et de la nourriture que s'exprime le blâme de la paresse et de l'oisiveté dans François le Champi :

[Blanchet] n'en fut que plus étranger aux profits d'argent, et, comme il engraissait, qu'il [...] n'aimait plus le travail, il chercha son aubaine dans des marchés de peu de foi et dans un petit maquignonnage d'affaires [...]. Sa concubine [...] l'emmenait dans les foires et assemblées pour tripoter dans des trigauderies et mener la vie de cabaret. Il apprit à jouer et fut souvent heureux; mais il eût mieux valu pour lui perdre toujours, afin de s'en dégoûter : car ce dérèglement acheva de le faire sortir de son $\operatorname{assiette}^{28}[\ldots]$.

La gourmandise se fait métaphore de la dégénérescence progressive des mœurs de Blanchet. Plus qu'une image: "hybris gastronomique ${ }^{29}$ " et déchéance morale deviennent synonymes. La gourmandise est, en outre, condamnable parce qu'elle est la manifestation extérieure d'une faiblesse mentale, d'une altération des facultés de jugement. Aussi George Sand déplore-t-elle qu'à Paris, lieu intense de sociabilité, elle " engraisse de corps et [...] maigri[t] d'esprit ${ }^{30}$ », formule annonçant déjà les reproches du muletier Huriel à Tiennet, lorsqu'il découvre les mœurs alimentaires des gens de la forêt et déplore leurs dimanches trop festifs devenant «une pesanteur de plus sur [leurs] estomacs et sur [leurs] esprits ${ }^{31}$ ». C'est que la «mollesse » et l'engraissement du

\footnotetext{
${ }^{26}$ George Sand, Correspondance, vol. III, à Maurice Dudevand, 8 juillet 1836, La Châtre, p. 464.

27 Idem.

${ }^{28}$ George Sand, François le Champi, Pierre Salomon et Jean Mallion (éd.), Paris, Classiques Garnier, 2014, chap. IV, p. 251, nous soulignons.

29 Olivier Assouly, Les Nourritures de Jean-Jacques Rousseau. Cuisine, goût et appétit, Paris, Classiques Garnier, 2016, p. 75.

30 George Sand, Correspondance, éd. citée, vol. V, à Hyppolite Chatiron, [Paris, 13 (?) mars 1841], p. 251.

31 George Sand, Les Maîtres sonneurs, éd. citée, Sixième veillée, p. 101 : «Le dimanche, accoudés sur des tables, mangeant plus que votre faim et buvant plus que votre soif, croyant vous divertir et vous
} 
corps sont les signes d'une dangereuse dépendance, comme le fait savoir la jeune mère à son fils :

Apprends à te préserver de la mollesse des habitudes de la vie matérielle, c'est-à-dire de la gourmandise, de la vanité, du libertinage, des vains amusements, des acquisitions inutiles et dispendieuses. [...] Fais-toi donc fort de corps et d'esprit. Faistoi expliquer ce que c'est que le stoïcisme. [...] Jamais un homme esclave de son ventre n'aura de dignité dans l'âme ${ }^{32}$.

La formule finale sera reprise presque mot pour mot dans La Daniella ${ }^{33}$, allégée des injonctions morales didactiques qui la précèdent. Aux yeux de l'auteur, la sobriété, voire l'austérité de l'existence, sont les gages d'une totale liberté à la fois du corps et de l'esprit. Aussi est-il nécessaire de savoir se priver. Au sein de ses fictions, la perspective de George Sand se fait christique : souffrir rend meilleur, épure l'âme et aiguise les facultés mentales. Et lorsque la continence est volontaire, pleinement choisie et assumée, celle-ci distingue les héros doués d'une exceptionnelle force de caractère, à l'instar de celle que possède Consuelo. Cette force est le signe d'élection des protagonistes aptes à être indépendants et autonomes :

Je connais la faim, et je sais y résister, malgré les éternels festins auxquels on voulait m'habituer à Riesenburg. Une journée est bientôt passée. Quand la chaleur sera venue, et mes jambes épuisées, je me rappellerai l'axiome philosophique que j'ai si souvent entendu dans mon enfance : «Qui dort dîne ${ }^{34}$.»

Consuelo se dégage définitivement du groupe des jeunes chanteuses frivoles de la scuola où elle a appris le chant, celles-ci se livrant, entre autres, au vain "plaisir de la gourmandise 35 ». Elle applique avec fermeté et courage ce "stoïcisme » que Sand conseille à Maurice. Libérée de toute entrave corporelle, elle existe en tant que sujet et peut mener à bien sa destinée. Cette grandeur apparaît d'autant plus significative que le siècle a vu émerger une bourgeoisie avide de jouissances matérielles et prompte à vivre de rentes.

\section{L'art d'être gourmand : des festins à partager}

Néanmoins, si l'on doit savoir se priver, l'on doit également savoir apprécier. Il est un plaisir de la table sandien et il existe une valorisation de la gourmandise chez l'auteur, une fois certaines conditions respectées. C'est ce qu'incline à penser l'évocation de son grand-oncle lors d'un repas familial dans Histoire de ma vie :

Il était gourmand, quoiqu'il mangeât fort peu, mais il avait une gourmandise sobre et de bon goût comme le reste, point fastueuse, sans ostentation, et qui se piquait même d'être positive ${ }^{36}$.

Quoiqu'avec une once d'ironie - parce que les « longs dîners servis, discutés, analysés et savourés avec tant de solennité [l]'ennuyaient mortellement »-, l'écrivaine dépeint avec bienveillance la passion gastronomique de son parent. Elle reconnaît même que «la

réconforter en vous indigérant, [...], vous faites, d'un jour de liesse et de repos, une pesanteur de plus sur vos estomacs et sur vos esprits [...]. »

32 George Sand, Correspondance, éd. citée, vol. III, à Maurice Dudevand, 8 juillet 1836, La Châtre, p. 464.

${ }^{33}$ George Sand, La Daniella, éd. citée, chap. XXXVI, p. 432, le narrateur affirme : « Je ne suis pas gourmand, et je ne comprends pas qu'un homme soit l'esclave de son ventre [...]. »

${ }^{34}$ George Sand, Consuelo, éd. citée, chap. LXIII, p. 409.

35 Ibid., chap. XI, p. 85.

${ }^{36}$ George Sand, Histoire de ma vie, éd. citée, vol. I, IIIe partie, chap. 2, p. 667. Idem pour la citation suivante. 
gourmandise exige un certain discernement ${ }^{37}$ ». Elle est admissible, voire souhaitable, pourvu que l'on en trace les contours éthiques. C'est ainsi moins la gourmandise que Sand condamne - puisqu'il s'agit d'un domaine où le « bon goût » peut s'inculquer -, que la gloutonnerie: l'excès. La gourmandise est une affaire de modération et de juste mesure : elle doit être rationnalisée. Dès lors, est-ce encore de la gourmandise ? En fait, Sand s'est ralliée à l'entreprise d'une partie de ses contemporains, qui tentent de racheter un terme longtemps dévalué en le régulant. Brillat-Savarin est de ceux-là parmi les premiers :

La gourmandise est une préférence passionnée, raisonnée et habituelle pour les objets qui flattent le goût.

La gourmandise est ennemie des excès ; tout homme qui s'indigère ou s'enivre, court risque d'être rayé des contrôles ${ }^{38}$.

George Sand apprécie ainsi, à titre personnel, les produits de qualité, et demande à maintes reprises à ses correspondants de lui en envoyer :

Parlons mangeaille, car, aussi bien, j'ai oublié de vous remercier de vos envois. Il paraît que c'est la mignonne Solange qui nous avait envoyé ces délicieux coquillages que nous nous arrachions honteusement Maurice et moi. Les grenades et le monstrueux tubercule [une pomme de terre] étaient également à se lécher les doigts [...]. Donc, [...] je vous ouvre un crédit, pour que vous nous fassiez un envoi considérable de coquillages, avec quelques patates et quelques grenades ${ }^{39}$.

L'auteur, non sans humour, a manifestement conscience de se laisser aller à un penchant gourmand condamnable a priori - mais auquel elle fait volontiers des entorses, ce dont témoignent les «festins de Lucullus ${ }^{40}$ » organisés sans complexe à Nohant ou sa participation aux dîners Magny ${ }^{41}$.

Dans une perspective déjà hygiéniste, George Sand valorise, d'une part, les "bonnes et saines friandises bien préférables aux bonbons ${ }^{42}$ ». Les aliments sont des remèdes et sa correspondance foisonne de références à tel ou tel régime en période de maladie, ainsi que de conseils prodigués à ses amis ${ }^{43}$. D'autre part, ce qu'elle blâme dans le comportement gourmand, c'est l'égoïsme, incarné par l'ex-gouvernante des Messieurs du Bois-Doré qui, "[n']étant plus forcée de savourer en secret les liqueurs et les friandises dérobées, [...] s'était livrée avidement à sa gourmandise ${ }^{44}$ » tandis que « [p]eu lui importait que vingt-cinq bons soldats [...] attendissent à la porte, le ventre creux ». $\mathrm{Au}$ sein d'une société encore profondément inégale, la gourmandise est inextricablement liée à la question socio-économique. En digne « fille de Jean-Jacques »

\footnotetext{
37 Ibid., vol. I, IVe partie, chap. 5, p. 1064.

38 Jean-Anthelme Brillat-Savarin, Physiologie du goût..., ouvr. cité, p. 270.

39 George Sand, Correspondance, éd. citée, vol. XV, à Charles Poncy, Nohant, 17 X bre [18]58, p. 218, Sand souligne.

40 Ibid., vol. XV, à Victor Borie, [Nohant, 15 décembre 1859], p. 606 ; ou vol. XX, à Lina Dudevand-Sand, [Paris], Lundi soir [10 février 1868], p. 701.

41 Anthony Glinoer et Vincent Laisney, L'Âge des Cénacles, confraternités littéraires et artistiques au XIX ${ }^{e}$ siècle, Paris, Fayard, 2013.

42 George Sand, Correspondance, éd. citée, vol. XXIII, à Antoine Scipion du Roure, Nohant, 30 décembre 1873, p. 648.

43 Tel est par exemple le cas lorsqu'elle préconise « une bonne nourriture succulente, [et] du vin de Bordeaux » à Charlotte Marliani pour favoriser son rétablissement (ibid., vol. V, à Charlotte Marliani, [Nohant, 3 juillet 1841], p. 348, Sand souligne).

44 George Sand, Les Beaux Messieurs de Bois-Doré, René Bourgeois (éd.), Grenoble, Éditions de l'Aurore, 1990, vol. 2, LIV, p. 111.
} 
réprouvant le luxe ${ }^{45}$, Sand juge la gourmandise humainement et politiquement indécente, ce qu'elle inculque à Maurice :

Jusqu'ici, je t'ai fourni d'argent, et je t'ai laissé libre d'acheter des friandises. [...] [S]onge à tous ces malheureux qui travaillent douze heures pour gagner dix sous ! Quand on y pense, les larmes en viennent aux yeux et les confitures semblent amères 46 .

La (bonne) nourriture doit, dans l'optique sandienne, faire l'objet de dons et de contre-dons : la gourmandise participe d'une fabrique du commun mise en place dans un «autour de la table» quotidiennement renouvelé. De ce point de vue, les agapes de Nohant ont tout d'une réminiscence des heureux banquets innocemment transgressifs de son enfance :

Fannelly [...] nous préparait sans cesse des surprises de gourmandise. C'était un melon magnifique, des gâteaux, des paniers de cerises ou de raisin, des beignets, des pâtés, que sais-je! [...] Pendant tout un été, nous ne fûmes nourries que par contrebande, et quelle folle nourriture ! [...] Mettre en commun nos friandises et les manger en cachette [...], c'était une fête, une partie fine et des rires inextinguibles ${ }^{47}$ [...].

L'on comprend ainsi qu'au sein des fictions sandiennes, le repas doive se partager : même les plus pauvres offrent volontiers de leur déjeuner aux compagnons d'infortune qu'ils croisent. Si gourmandise il y a, elle n'a de sens qu'au sein de la communauté, ce qu'exprime le "Grand-Bûcheux » dans Les Maîtres sonneurs: "il ne sera pas dit qu'on mangera le rôti et boira le vin de Sancerre à la loge du Grand-Bûcheux sans que tous ses amis y aient part ${ }^{48} »$. Mais dans le roman sandien, la gourmandise est supplantée par le don : peu importent la qualité et la quantité des mets, pourvu qu'ils soient mis en commun. Plus que l'art d'être gourmand, c'est l'art de manger ensemble qui intéresse George Sand, parce qu'il participe de l'utopie républicaine et sociale. Lorsque Consuelo rencontre le jeune Hyndn, celui-ci est prêt de se vexer en voyant que la jeune fille s'apprête à ne pas honorer sa générosité : «[...] êtes-vous trop fière pour accepter de moi un pauvre morceau de pain? Hélas ! vous voyez, je n'ai que cela à vous offrir ${ }^{49}$. » Et Consuelo,

[...] sentant bien que ce serait mal reconnaître l'élan fraternel de son amphitryon que de ne pas manger en sa compagnie, [...] se rassit non loin de lui, et se mit à dévorer ce pain, au prix duquel les mets les plus succulents qu'elle eût jamais goûtés à la table des riches lui parurent fades et grossiers.

Les compagnons redonnent sa force étymologique au terme (formé de cum et panis, désignant « celui avec qui l'on partage le pain »), annonçant déjà le binôme uni et soudé qu'ils viendront à former. La nourriture a toutes ses lettres de noblesse lorsque, d'une part, elle soulage la faim, et quand, d'autre part, elle scelle une relation fraternelle. Il n'est ainsi pas anodin que ce soit du pain que les protagonistes rompent ensemble: Consuelo et son comparse rejouent la Cène christique sur un mode laïc et républicain ${ }^{50}$.

\footnotetext{
45 Voir Olivier Assouly, Les Nourritures de Jean-Jacques Rousseau.., ouvr. cité.

46 George Sand, Correspondance, vol. III, à Maurice Dudevand-Sand, [La Châtre, 28 juin 1836], p. 452.

${ }^{47}$ George Sand, Histoire de ma vie, éd. citée, vol. I, III ${ }^{e}$ partie, chap. 13, p. 938, nous soulignons la dernière expression.

48 George Sand, Les Maîtres sonneurs, éd. citée, Treizième veillée, p. 189.

${ }^{49}$ George Sand, Consuelo, éd. citée, chap. LXIV, p. 411-412. Idem pour la citation suivante.

$50 \mathrm{Au}$ sujet de la récupération républicaine du Christ par les Romantiques, voir Frank Paul Bowman, $L e$ Christ romantique, Genève, Librairie Droz, 1973 ; et Le Christ des barricades : 1789-1848, Paris, Éditions du Cerf, 1987.
} 
$\mathrm{Si}$ « le pain [est] le plus pur des aliments ${ }^{51}$ » pour le meunier d'Angibault, c'est sans nul doute parce qu'il est le plus aisément partagé : il s'agit de la nourriture démocratique par excellence.

À la lumière des fictions sandiennes déclinant la nourriture sous tous ses aspects - rabelaisiens, politiques, culturels et même ethnologiques -, la vie à Nohant s'éclaire d'un jour nouveau : la gourmandise bien pensée, modérée, non nécessaire mais surtout partagée au sein d'une communauté prompte à toujours s'agrandir constitue in fine le fondement d'une forme de résistance morale et politique face aux mœurs décadentes de la capitale, à la propagation du mauvais goût et à la montée de l'individualisme. Dans un siècle qui a tant mangé52, George Sand a élaboré sa propre recette : loin des festins littéraires satiriques d'un Zola - songeons à l'oie dépecée avidement à la table de Gervaise dans L'Assommoir - ou d'un Flaubert - dépeignant complaisamment le repoussant spectacle du duc de Laverdière en train de manger dans Madame Bovary -, elle est aux antipodes également de la célébration sensuelle d'un Dumas alléché par le "pied d'éléphant» ou les "pattes d'ours » dans son Grand dictionnaire de cuisine œuvre somme hymne à la créativité dans tous les domaines -, ou des repas dandy du Baudelaire de Fanfarlo. Devenue éthique, la gourmandise sandienne a le goût d'une utopie politique: elle doit se concevoir comme un art de bien manger ensemble, satisfaisant un appétit plus relationnel et social que corporel. Elle est, à ce titre, de ces « jouissances » dont l'homme ne «doit» pas se retirer, pourvu qu'il soit parvenu à la rendre « noble».

Amélie CALDERONE

CNRS-IHRIM

51 George Sand, Le Meunier d'Angibault, Marielle Caors (éd.), Grenoble, Éditions de l'Aurore, 1990, chap. XXIII, p. 173.

${ }^{52}$ Voir Jean-Paul Aron, Le Mangeur du XIXe siècle, Paris, Les Belles Lettres, 2013. 


\section{Résumés}

George Sand n'a jamais écrit de traité spécifiquement consacré à la gourmandise. Néanmoins, ses œuvres et ses textes personnels regorgent de mentions culinaires et de personnages gourmands, au travers desquels se dessine un imaginaire sandien de la gourmandise, non exempt de certains clichés. Le rapport à la nourriture permet dès lors de repenser la question de l'idéalisme sandien, qui doit se concevoir comme alimentation de la matérialité par l'idée. Si elle réprouve, sur le plan individuel, un penchant dont elle fait l'une des causes des déchéances physique et morale et de la perte de la pleine liberté d'esprit, elle accepte cependant une gourmandise, pourvu qu'elle soit rendue éthique. Modérée, de bon goût, non nécessaire et partagée, celle-ci peut même devenir, à l'échelle collective, l'occasion d'une célébration de l'utopie fraternelle républicaine.

George Sand never wrote a treatise specifically dedicated to greed. Nevertheless, her works and personal texts are full of culinary images and gourmet characters that constitute a Sandian imagination of greed, albeit one not exempt from some stereotyping. The relationship with food therefore makes it possible to rethink the question of Sandian idealism, which must be conceived as the nourishment of materiality by the idea. If she disapproves, on an individual level, of a proclivity she equates with physical and moral decay and the loss of freedom of spirit, she nevertheless accepts gluttony, provided that it is made ethical. Moderate, of good taste, unnecessary and shared, it can even become, in a collective sense, a celebration of republican utopia. 


\section{Bibliographie}

\section{Critique}

ARON Jean-Paul, Le Mangeur du XIXe siècle, Paris, Les Belles Lettres, 2013.

ASSOULY Olivier, Les Nourritures de Jean-Jacques Rousseau. Cuisine, goût et appétit, Paris, Classiques Garnier, 2016.

BOWMAN Frank Paul, Le Christ des barricades : 1789-1848, Paris, Éditions du Cerf, 1987. BOWMAN Frank Paul, Le Christ romantique, Genève, Librairie Droz, 1973.

GLINOER Anthony et LAISNEY Vincent, L’Âge des Cénacles, confraternités littéraires et artistiques au XIXe siècle, Paris, Fayard, 2013.

SCHOR Naomi, George Sand and idealism, New-York, Columbia University Press, 1993.

ZANONE Damien (dir.), George Sand et l'idéal. Une recherche en écriture, Paris, Honoré Champion, coll. « Littérature et Genre », 2017.

QEuvres personnelles de George Sand

SAND George, Correspondance, Georges Lubin (éd.), Paris, Garnier, coll. « Classiques Garnier », 25 vol., 1964-1991 ; vol. 26, Tusson, Du Lérot, 1995.

SAND George, Histoire de ma vie, dans CEuvres autobiographiques, George Lubin (éd.), Paris, Gallimard, coll. « Bibliothèque de la Pléiade », 2 vol., 1970 et 1971.

QEuvres fictionnelles de George Sand

SAND George, Les Beaux Messieurs de Bois-Doré, René Bourgeois (éd.), Grenoble, Éditions de l'Aurore, 2 vol., 1990.

SAND George, Consuelo. La Comtesse de Rudolstadt, Damien Zanone (éd.), Paris, Robert Laffont, coll. «Bouquins », 2004.

SAND George, La Daniella, Alex Lascar (éd.), Paris, Honoré Champion, 2016.

SAND George, François le Champi, Pierre Salomon et Jean Mallion (éd.), Paris, Classiques Garnier, 2014.

SAND George, Les Maîtres sonneurs, Pierre Salomon et Jean Mallion (éd.), Paris, Classiques Garnier, 2014.

SAND George, Le Meunier d'Angibault, Marielle Caors (éd.), Grenoble, Éditions de l'Aurore, 1990.

SAND George, Monsieur Sylvestre, Paris, Michel Lévy frères, 1866.

SAND George, La Petite Fadette, Pierre Salomon et Jean Mallion (éd.), Paris, Classiques Garnier, 2014.

SAND George, Teverino, dans Vies d'artistes, Marie-Madeleine Fragonard (éd.), Paris, Omnibus, 2004.

Recueils de textes de George Sand

SAND Christine, À la table de George Sand, Paris, Flammarion, 1987.

SAND George, Scènes gourmandes, repas et recettes du Berry, Béatrice Didier (éd.), Paris, EJL, 1999. 


\section{Ouvrages contemporains}

BRILLAT-SAVARIN Jean-Anthelme, Physiologie du goût ou Méditations de gastronomie transcendante, Paris, Sautelet, 1826.

LAROUSSE Pierre, Grand Dictionnaire universel $d u$ XIXe siècle, 17 vol., Paris, Administration du Grand Dictionnaire Universel, 1866-1877. 